\title{
Should We Fiddle with Gut Microbiome in Critically Ill?
}

\author{
Balaji Venkatachalam ${ }^{1}$, Babu Kuruvilla Abraham²
}

\begin{abstract}
The gut that we took for granted in the critically ill, as just a conduit for food passage has over the decade or so shown us that it is an active endocrine and exocrine organ with over 40 trillion microorganisms living commensally within it. This cosmos of microorganisms that is called the gut microbiome comprises roughly 1,000 different species and put together is more DNA than the entire human genome. Under normal circumstances, in a healthy individual multiple elements of the gut viz intestinal epithelium, gut barrier function, the microbiomes, all put together offer protection against infection and this is crucial in maintenance of health. Any change to the norm, be it in the form of surgical interventions, the introduction of medications, or the pathophysiological effects of systemic disease leads to a $360^{\circ}$ alteration in this finely construed ecosystem leading to devastating effects that go beyond the boundaries of the gut itself. Intestinal epithelium helps to absorb nutrients as well as acts as the coordinator of mucosal immunity (first line of immune defense). During ill health, gut epithelial apoptosis occurs, alterations happen in the tight epithelial junctions leading to loss of gut barrier function and loss of the mucosal immunity leading to mucosal damage and hyperpermeability. Lastly, the microbiome is transformed into a pathobiome, with resultant increase in pathogenic bacteria and induction of virulence in commensal gut bacteria. Multiple organ damage starts to set in, caused by toxins leaving the intestine via both portal blood flow and mesenteric lymph. This review article traces the gut microbiomic ecology in health and sickness, modern tools that are used to manipulate gut microbiome in the search for the prevention and treatment of critical illness and will explore if appropriate manipulation of gut microbiome can influence or modulate the course of critical illness.
\end{abstract}

Keywords: Dysbiosis, Fecal microbial transplantation, Gut-lung axis, Microbiome, Pathobiome, Probiotics, Synbiotics.

Indian Journal of Critical Care Medicine (2020): 10.5005/jp-journals-10071-23627

\section{INTRODUCTION}

The gut that we took for granted in the critically ill, as just a conduit for food passage has over the decade or so shown us that it is an active endocrine and exocrine organ with over 40 trillion microorganisms living commensally within it. This cosmos of microorganisms that are called the gut microbiome comprises roughly 1,000 different species and put together is more DNA than the entire human genome. Under normal circumstances, in a healthy individual multiple elements of the gut viz intestinal epithelium, gut barrier function, the microbiomes, all put together offer protection against infection and this is crucial in maintenance of health. Any change to the norm, be it in the form of surgical interventions, the introduction of medications, or the pathophysiological effects of systemic disease leads to a $360^{\circ}$ alteration in this finely construed ecosystem leading to devastating effects that go beyond the boundaries of the gut itself. Intestinal epithelium helps to absorb nutrients as well as acts as the coordinator of mucosal immunity (first line of immune defense). During ill health, gut epithelial apoptosis occurs, alterations happen in the tight epithelial junctions leading to loss of gut barrier function and loss of the mucosal immunity leading to mucosal damage and hyperpermeability. Lastly, the microbiome is transformed into a pathobiome, with resultant increase in pathogenic bacteria and induction of virulence in commensal gut bacteria. Multiple organ damage starts to set in, caused by toxins leaving the intestine via both portal blood flow and mesenteric lymph.

This review article traces the gut microbiomic ecology in health and sickness, modern tools that are used to manipulate gut microbiome in the search for the prevention and treatment of critical illness and will explore if appropriate manipulation of gut microbiome can influence or modulate the course of critical illness.

\footnotetext{
1,2Department of Critical Care Medicine, Apollo Hospitals, Chennai, Tamil Nadu, India
}

Corresponding Author: Balaji Venkatachalam, Department of Critical Care Medicine, Apollo Hospitals, Chennai, Tamil Nadu, India, Phone: +91-44-33151510, e-mail: balajivenkatachalamcritic@gmail. com

How to cite this article: Venkatachalam B, Abraham BK. Should We Fiddle with Gut Microbiome in Critically III? Indian J Crit Care Med 2020;24(Suppl 4):S211-S214.

Source of support: Nil

Conflict of interest: None

\section{The "Normal Gut" and "Gut in the NORMAL"}

A single-cell layer epithelium of $30 \mathrm{~m}^{2}$ covering the gut plays a pivotal role in host homeostasis, acts as a first-line immune defense, coordinates with gut-associated lymphoid tissue, secreting hundreds of cytokines, hormones, and antimicrobial peptides. ${ }^{1}$ The gut is a continually renewing organ with a turnover time of 1 week with a fine balance between apoptosis-anti-apoptosis factors. The complex intestinal immune system has an extremely diverse ecosystem having more lymphocytes than any other organ. Microbiota is an umbrella term to include all the microbes in a population, including bacteria, archaea, and eukarya. Most studies look at the bacterial microbiota in preference, because of its abundance and diversity. The collective genetic content of the microbiota is referred to as the microbiome. 
The crosstalk between mucosal immune system and microbiome regulates the release and utility of CD4 cells, antimicrobial peptides, secretive IgA, innate lymphoid cells, mucosa-associated invariant T cells, and cells of the mononuclear phagocyte systems, maintaining the overall homeostasis of all organ systems. Modern, culture-independent techniques, such as, $16 \mathrm{~S}$ ribosomal RNA ( $r R N A$ ) and shotgun metagenomic sequencing, have established the important role of microbiome in metabolism, development of the immune system, and host defense against pathogens.

\section{The "Critically Ill Gut" and "Gut in Critically Ill"}

\section{Intestinal Hyperpermeability}

Any alteration in intestinal integrity or microbiome worsens sepsis and vice versa, i.e., sepsis further propagates gut damage. This leads to a downward spiral in a progressive feedback fashion.

Sepsis leads to an upregulated apoptosis, profound decrease in crypt proliferation, and decrease in villus length. ${ }^{2}$

The structural changes induced by sepsis are seen as early as 1 hour of its onset and continues for up to 48 hours, resulting in loss of tight junctions, impaired barrier function, loss of hydrophobicity of the mucus layer, and ultimately, intestinal hyperpermeability.

\section{Pathobiome}

Both the composition and the density of microbiome play a role in maintaining normal homeostasis. Sepsis alters both the aspects, within hours of onset. Emerging data clearly point toward the link between sepsis and transformation of "health inducing microbiome" to "disease promoting pathobiome".

The largest study (American Gut Project) examined microbiota in the skin, tongue, and stool of 115 critically ill patients, less than 48 hours of intensive care unit (ICU) admission and ICU discharge or 10th ICU day, with over 1,000 patients. ${ }^{3}$ The study showed significant dysbiosis progression in all the anatomical areas.

The emergence of pathobiome in sepsis is multifactorial. The causes include newly acquired genes by microbiome, treatment interventions in ICU, drugs (antibiotics, proton-pump inhibitors, and opioids), components of feeding (carbohydrates, lipid, and protein), and route (enteral/parenteral).

The gut microbiota of critically ill patients is characterized by reduced diversity, reduced abundance of key commensals (such as, Faecalibacterium, Blautia, Ruminococcus), and overgrowth of one species, such as, Escherichia, Salmonella, Shigella, Clostridium difficile, Enterococcus, or Staphylococcus.

\section{Gut-Lymph/Gut-Lung Axis}

Many preclinical and clinical data reveal that gut is the source of lung bacteria and overall sepsis. The releases of toxins from the diseased gut travels through lymph as well as through blood and reach distant organs. ${ }^{4}$

The clinical trials, society guidelines, and articles have largely ignored one of the largest organs of human body, the gut microbiome. Conditions like septic shock, acute respiratory distress syndrome (ARDS), acute kidney injury (AKI), and multiorgan dysfunction syndrome (MODS) cause tremendous global mortality, morbidity, and economic burden. In spite of that, we only have supportive therapies for these conditions and no targeted treatment plan is available. One rational explanation for not having a targeted therapy is that the host inflammation and cellular injury are just downstream consequences of an ignored or forgotten upstream source: Neglected or mismanaged microbiome. The altered microbiome has negative ripple effects on all organ systems.

\section{Biological Catastrophe of Critical ILLness}

As early as 1969, Johanson et al. observed that body's microbiome is heavily altered by critical illness. ${ }^{5}$ Not all hospitalized patients, but only critically ill patients have drastic change in ecology of microbiome. The change in microbiota [with increase in gramnegative bacteria (GNB)] depends more on the severity of patient's illness, not just on their location in the hospital.

The composition of microbiome is determined by three key ecological factors: Entry of new microbes into the community, elimination of established or commensal microbes from the community, and relative multiplication rates of individual microbes. The gut and lung ecosystems undergo drastic changes in all the three above factors, not just because of the pathophysiological effects of critical illness but also because of the interventions performed in ICU.

\section{Ecological Effects of Critical Illness on Gut Microbiome ${ }^{6-9}$}

\begin{tabular}{ll}
\hline Pathophysiological effects & Clinical interventions \\
\hline Altered oropharyngeal & Gastric acid suppression (PPI) \\
microbiota & Supine position \\
Poor oral intake & Parenteral antibiotics \\
Poor gut motility & Parenteral feeding \\
Impaired gut mucosal integrity/ & Oral decontamination (topical \\
ischemia & chlorhexidine) \\
Hyperglycemia/hypokalemia & Selective decontamination of \\
Reduced bile salt concentration & digestive tract \\
Endogenous opioid production & Sedation, opiates, neuromuscu- \\
Increased catecholamine and & lar blockers \\
cytokine production & Systemic catecholamines \\
Impaired mucosal immunity & \\
(decreased IgA) & \\
\hline
\end{tabular}

In healthy individuals, the primary means of microbial elimination from the gut microbiome is through defecation. A healthy adult expels about $10^{14}$ bacterial cells/day. In critically ill patients, transit time is substantially slowed by various pathophysiological (glucose and electrolyte disturbances and endogenous opioid production) and therapeutic (sedatives, opiates, and systemic catecholamines) factors. Keeping the patient nil per oral (NPO) has its own negative consequences. When the gut is not utilized for feeding, mucosal integrity is lost by ischemia, resulting in bacterial translocation.

In the large intestine, the microbiota ferments non-digested dietary fibers and dietary and host-derived proteins. The fermentation of fibers leads to production of butyrate, which is used by epithelial cells as an energy source. This energy supply is interrupted in parenterally fed patients. The clinical relevance of these effects is still in its infancy and more clinical studies are needed to elucidate its effects. 


\section{Altered Microbiome of Injured Alveolus}

Healthy lungs are not necessarily sterile as previously thought and bacteria can be cultured from them. The lungs get exposed to various pathogens not just from upper respiratory tract but also through the blood.

Healthy lungs receive most of the bacteria from the oral microbiota. That is why the lung microbiota largely resembles the microbiota of the oropharynx (Veillonella and Prevotella), and not that of the gastrointestinal tract. During critical illness, the oropharynx becomes overpopulated with pathogenic organisms, such as, Pseudomonas aeruginosa and Klebsiella pneumoniae. Also, the gut becomes the primary source of bacterial migration to the lung. This results in complete alteration of lung microbiome in critically ill.

But, the normal alveolar space is relatively inhospitable for most bacteria as it lacks the nutrient substrate necessary for bacterial reproduction. ${ }^{10}$ Also, the bidirectional movement of the lungs and aerobic environment adds to this protection.

The gut environment provides various protein and carbohydrate sources for the bacteria to thrive, but the healthy alveolar epithelium is lined with lipid rich, bactericidal surfactant. But, when the alveoli are injured as in ARDS or pneumonia, the previously empty alveoli are filled with protein rich fluid which acts as a nutrition for flooding bacteria. The loss of protective surfactant coupled with impaired mucociliary clearance makes the microbial environment of lung very similar to that of gut. Also, increased catecholamine concentrations result in change in microbiome and the emergence of one dominant species. The direct and indirect lung injuries provoke a cascade of alveolar inflammation, which results in catecholamine surge and further dysbiosis. It is worthy to note that most pathogens that arise in critical illness are of enteric origin. There exists a dysregulated feedback between microbiome and alveolar injury, resulting in worsening inflammation, alveolar edema, and further dysbiosis, which forms the central theme of sepsis. ${ }^{11}$

The gut-lung axis is a two-way street. Intestinal microbiome influences the lung health and vice versa. For example, murine models show that pulmonary infections with tuberculosis, influenza, and Burkholderia pseudomallei influence the composition of the gut microbiome.

\section{Analysis of Microbiome}

Non-culture methods are increasingly becoming popular to detect and analyze microbiome. One example is the 16S rRNA genetic marker which is relatively easy to amplify. With modern sequencing techniques, in-depth, high-resolution analysis of microbial environments is possible. ${ }^{12}$

Techniques like metagenomics (determination of the collective genomes and functional capacity of all the members present in a microbial community), metaproteomics (analysis of difference in the amount of proteins expressed and the proteins predicted by metagenomics), metatranscriptomics (elucidation of the specific functional roles microbes) are getting refined and expected to be available for clinical use soon. Together, they develop and generate complex data sets about microbial functions, which may alter the way critical interventions are practiced in ICU.

Recent advancements in the successful culture of the previously "difficult to culture" human microbiota have revealed a whole spectrum of novel bacterial species and a chance to understand their complicated system much better. ${ }^{13}$

\section{Therapeutic Approaches Targeting THE GUT}

The gut epithelium, mucus, and its permeability are potential therapeutic targets in critically ill patients, but no such therapy exists at the bedside for a clinician. However, many preclinical strategies like epidermal growth factor (EGF), membrane permeable inhibitor of MLCK, mucus surrogates, and vagus nerve agonists are actively researched to manipulate gut apoptosis, proliferation, and permeability. ${ }^{14}$

Another conceptual target is to address bacterial virulence factors by promoting "healthy" bacteria and decreasing "unhealthy" bacteria, by the use of probiotics (exogenous bacteria) as well as fecal microbial transplantation (FMT).

By definition, probiotics (Lactobacillus, Bifidobacterium) are living strains of bacteria that add the population of "healthy" bacteria in the gut. Prebiotics are specific plant fibers that act as food or fuel for healthy bacteria in the gut, thereby promoting their growth. Synbiotics refer to food supplements combining the benefits of probiotics and prebiotics in the form of synergism.

Meta-analyzes regarding probiotics have shown a decrease in ventilator-associated pneumonia, without alterations in mortality or length of stay. However, the evidence is not high quality as the studies had significant heterogeneity in terms of types of bacteria used and timing of administration. ${ }^{15}$ More studies are needed to elucidate the benefits of probiotics in critically ill.

A recent randomized control trial (RCT) from India, with 4,556 healthy infants showed that the oral administration of Lactobacillus plantarum in combination with fructooligosaccharide in the first week of life could reduce the incidence of sepsis in the first 60 days of life. ${ }^{16}$

Synbiotics may work as an adjunctive therapy to prevent surgical site infections and sepsis among adult surgical patients. ${ }^{17}$

Experimental exposure to toll-like receptor (TLR) agonists and nucleotide-binding oligomerization domain (NOD)-like receptors in the intestine, by substances, such as, lipopolysaccharide (LPS), peptidoglycan, and lipoteichoic acid, improve the lungs ability to clear bacteria. ${ }^{18}$ These observations suggest that the intestinal microbiota is important for alveolar health.

Fecal microbial transplantation is an attempt to replenish normal bacterial flora in the diseased gut in which the entire microbiome is transplanted from a healthy donor. One clinical area where FMT has shown reasonable success is in the management of recurrent $C$. difficile infection ( $92 \%$ response rate). In inflammatory bowel disease, FMT has shown some promise. To date, data on FMT in the ICU are limited to case reports only. As such, FMT should be considered experimental in critical illness currently, as its safety and efficacy need to be systematically studied.

Selective decontamination of the digestive tract (SDD) targets pathogenic gut bacteria and is diametrically opposite to probiotics and FMT. In areas with low antimicrobial resistance, SDD has shown reduced mortality in multiple studies and meta-analyzes. There are theoretical concerns that SDD could induce multidrug resistance. A recent study randomized with around 8,000 patients on mechanical ventilation in 13 ICUs with high-moderate levels of antibiotic resistance to (a) a modified version of SDD (without a 
4-day course of intravenous antibiotics), (b) selective oropharyngeal decontamination, and (c) chlorhexidine mouthwash and compared them to a baseline period. No reduction in mortality or ICU-acquired bloodstream infection was demonstrated in any of the groups compared to baseline.

In the last decade, researchers have shown great interest in therapeutic strategies to prevent induction of new virulence factors in bacteria. Bacterial sensing of intraluminal phosphate, and deficiency of phosphate, plays a critical role in the induction of virulence. Repletion of intraluminal (not intravenous) phosphate may trick bacteria into "believing" that a diseased host is healthy. Murine data and preclinical studies show that enterally administered polyethylene glycol-conjugated phosphate improves survival in murine intra-abdominal sepsis, which may be extrapolated to humans. More studies are needed to confirm this. ${ }^{19}$

\section{Conclusion}

The importance of the gut microbiome is often overlooked in critically ill patients. The microbiome in critically ill patients is influenced by their illness, the care provided and is characterized by lower diversity, lower abundance of key commensal bacteria and abnormal overgrowth by one species. These changes in microbiota can adversely affect the prognostic outcomes in critically ill patients. The gut-lung axis signals are bidirectional and microbiome alterations in one area can adversely affect the other. More research with clinical treatment targets are needed to address this important area of critical care.

\section{References}

1. Birchenough GM, Johansson ME, Gustafsson JK, Bergstrom JH, Hansson GC. New developments in goblet cell mucus secretion and function. Mucosal Immunol 2015;8(4):712-719. DOI: 10.1038/ mi.2015.32.

2. Otani S, Coopersmith CM. Gut integrity in critical illness. J Intensive Care 2019;7(1):17. DOI: 10.1186/s40560-019-0372-6.

3. McDonald D, Ackermann G, Khailova L, Baird C, Heyland D, Kozar R, et al. Extreme dysbiosis of the microbiome in critical illness. $\mathrm{mSphere}$. 2016;1(4):e00199-16. DOI: 10.1128/mSphere.00199-16.

4. Dumas A, Bernard L, Poquet Y, Lugo-Villarino G, Neyrolles O. The role of the lung microbiota and the gut-lung axis in respiratory infectious diseases. Cell Microbiol 2018;20(12):e12966. DOI: 10.1111/cmi. 12966.

5. Johanson WG, Pierce AK, Sanford JP. Changing pharyngeal bacterial flora of hospitalized patients. Emergence of gramnegative bacilli. N Engl J Med. 1969;281(21):1137-1140. DOI: 10.1056/ NEJM196911202812101.

6. David LA, Maurice CF, Carmody RN, Gootenberg DB, Button JE, Wolfe $B E$, et al. Diet rapidly and reproducibly alters the human gut microbiome. Nature 2014;505(7484):559-563. DOI: 10.1038/ nature12820.

7. Björnsson ES, Urbanavicius V, Eliasson B, Attvall S, Smith U, Abrahamsson $\mathrm{H}$. Effects of hyperglycemia on interdigestive gastrointestinal motility in humans. Scand J Gastroenterol 1994;29(12):1096-1104. DOI: 10.3109/00365529409094894.

8. Deitch EA. Gut-origin sepsis: evolution of a concept. Surgeon 2012;10(6):350-356. DOI: 10.1016/j.surge.2012.03.003.

9. Zaborin A, Smith D, Garfield K, Quensen J, Shakhsheer B, Kade M, et al. Membership and behavior of ultra-low-diversity pathogen communities present in the gut of humans during prolonged critical illness. MBio 2014;5(5):e01361-14. DOI: 10.1128/mBio.01361-14.

10. Venkataraman A, Bassis CM, Beck JM, Young VB, Curtis JL, Huffnagle $\mathrm{GB}$, et al. Application of a neutral community model to assess structuring of the human lung microbiome. MBio 2015;6(1):e01163-15. DOI: 10.1128/mBio.02284-14.

11. Dickson RP, Erb-Downward JR, Huffnagle GB. Towards an ecology of the lung: new conceptual models of pulmonary microbiology and pneumonia pathogenesis. Lancet Respir Med 2014;2(3):238-246. DOI: 10.1016/S2213-2600(14)70028-1.

12. Michael Janda J, Abbott SL. 16S rRNA gene sequencing for bacterial identification in the diagnostic laboratory: pluses, perils, and pitfalls. J Clin Microbiol 2007;45(9):2761-2764. DOI: 10.1128/JCM. 01228-07.

13. Browne HP, Forster SC, Anonye BO, Kumar N, Neville BA, Stares MD, et al. Culturing of 'unculturable' human microbiota reveals novel taxa and extensive sporulation. Nature 2016;533(7604):543-546. DOI: 10.1038/nature17645.

14. Klingensmith NJ, Yoseph BP, Liang Z, Lyons JD, Burd EM, Margoles $L M$, et al. Epidermal growth factor improves intestinal integrity and survival in murine sepsis following chronic alcohol ingestion. Shock 2017;47(2):184-189. DOI: 10.1097/SHK.0000000000000709.

15. Manzanares W, Lemieux M, Langlois PL, Wischmeyer PE. Probiotic and synbiotic therapy in critical illness: a systematic review and meta-analysis. Crit Care 2016;19(1):262. DOI: 10.1186/s13054-0161434-y.

16. Panigrahi P, Parida S, Nanda NC, Satpathy R, Pradhan L, Chandel $D S$, et al. A randomized synbiotic trial to prevent sepsis among infants in rural India. Nature 2017;548(7668):407-412. DOI: 10.1038/ nature23480.

17. Kasatpibal N, Whitney JD, Saokaew S, Kengkla K, Heitkemper MM, Apisarnthanarak A. Effectiveness of probiotic, prebiotic, and synbiotic therapies in reducing postoperative complications: a systematic review and network meta-analysis. Clin Infect Dis 2017;64(Suppl. 2):S153-S160. DOI: 10.1093/cid/cix114.

18. Budden KF, Gellatly SL, Wood DL, Cooper MA, Morrison M, Hugenholtz $P$, et al. Emerging pathogenic links between microbiota and the gut-lung axis. Nat Rev Microbiol 2017;15(1):55-63. DOI: 10.1038/ nrmicro.2016.142.

19. Zaborin A, Defazio JR, Kade M, Kaiser BL, Belogortseva N, Camp DG, et al. Phosphate-containing polyethylene glycol polymers prevent lethal sepsis by multidrug-resistant pathogens. Antimicrob Agents Chemother 2014;58(2):966-977. DOI: 10.1128/AAC.02183-13. 